\title{
The effect of perturbations on the circular restricted four-body problem with variable masses
}

\author{
Abdullah A. Ansari, Ziyad A. Alhussain, Rabah Kellii* \\ College of Science at Al-Zulfi, Majmaah University, KSA.
}

\begin{abstract}
This paper presents a new investigation of the circular restricted four body problem under the effect of any variation in coriolis and centrifugal forces. Here, masses of all the bodies vary with time. This has been done by considering one of the primaries as oblate body and all the primaries are placed at the vertices of a triangle. Due to the oblateness, the triangular configuration becomes an isosceles triangular configuration which was an equilateral triangle in the classical case. After evaluating the equations of motion, we have determined the equilibrium points, the surfaces of the motion, the time series and the basins of attraction of the infinitesimal body. We note that, when we increase both the coriolis and centrifugal forces, the curves, surfaces of motion, and the basins of attraction are shrinking except when we fix the centrifugal force and increase the value of coriolis force, the curves are expanding and the equilibrium points are away from the origin. The behavior of the surfaces of motion and the basins of attraction in the last case (fixing the centrifugal force and increasing the value of coriolis force) will be studied next. In all the present study, we found that all the equilibrium points are unstable. (C)2017 All rights reserved.
\end{abstract}

Keywords: Circular restricted four body problem, isosceles triangular configuration, coriolis and centrifugal forces, oblateness, variable mass, basins of attraction, unstable.

2010 MSC: 70F15, 85A20, 85A99, 70F05, 70F07.

\section{Introduction}

Since many decades scientists performed many mathematical models in the celestial mechanics as twobody, three-body, four-body, and n-body problems. The restricted problem was also an interesting topic for them. Many scientists have studied the restricted three-body and the restricted four-body problems. Many related topics have been studied. For example, in [39], Moulton evaluated the equations of motion in the four-body problem and demonstrated that the finite bodies can be placed by twenty-eight different ways. On the other hand, Jeans in [27] discussed the two-body problem with variable mass and Meščerskiı in [37] investigated the mechanics of the bodies with variable mass. For their work, Sharma et al. in [45] investigated numerically the location of the collinear libration points in the restricted three-body problem when the primaries are oblate spheroids and observed that these equilibrium points are unstable. To be complete, we give below an important list of works close to our investigation.

\footnotetext{
${ }^{*}$ Corresponding author

Email addresses: a.ansari@mu.edu.sa (Abdullah A. Ansari), z.alhussain@mu.edu.sa (Ziyad A. Alhussain), r.kellil@mu.edu.sa (Rabah Kellil)

doi:10.22436/jmcs.017.03.03
} 
In [13], Bhatnagar et al. examined the stability of libration points in the restricted problem with the perturbations in the coriolis and centrifugal forces and found that there is no effect of perturbations on the collinear points and they remain unstable, however the range of the stability for the equilateral triangular points increases or decreases due to the perturbations. Simo in [49] discussed the solutions for arbitrary masses and the manifolds of degeneracies in the restricted four-body problem. They have examined the linear stability in the restricted and general cases. Majorana in [34] examined the linear stability of eight equilibrium points which depends on the values of the mass parameter in the restricted four-body problem. Shrivastava et al. in [47] deduced the equations of motion in the restricted three-body problem with decreasing mass by using the Jeans law and Meshcherskii transformation. Shrivastava et al. in [46] evaluated the equilibrium points in the Robes restricted problem of three-bodies with effect of perturbations in the coriolis and centrifugal forces. Singh et al. in [50-59] studied the restricted problem of three-bodies and four-bodies in circular and elliptic cases with different perturbations. Khanna et al. in $[29,30]$ explored the existence and stability of libration points in the restricted three-body problem when the smaller primary is a triaxial rigid body and the bigger one an oblate spheroid and observed that there are five libration points in which three collinear libration points are unstable and two triangular points are stable for the particular mass parameter. They also observed that the triangular points have long and short periodic elliptical orbits for the same mass parameter. Hallan et al. in $[24,25]$ investigated the locations, linear stability, and non-linear stability of the equilibrium points in the Robe's circular restricted three-body problem with the effect of perturbations in coriolis and centrifugal forces. They found that the locations of equilibrium points are not affected by coriolis force and the range of stability increases or decreases depending upon the perturbations. Shu et al. in [48] investigated the existence of libration points and their linear stability under the effects of coriolis and centrifugal forces in the Robe's restricted three-body problem and found that the linear stability depends on the parameter $k$. Leandro in [32] investigated the bifurcations of central configurations by the uniqueness of convex central configurations up to a symmetry in the planar restricted four-body problem. They have discussed also the spectral stability and their bifurcations with all the possible configurations. AbdulRaheem et al. in [1,2] studied the stability and periodic orbits of equilibrium points in the restricted three-body problem under the effect of radiation, oblateness, and perturbing forces. They observed that the perturbing forces affect the stability, period, orientation, and the eccentricities of the long and short periodic orbits. Lukyanov in [33] investigated the close binary stars with transfer since Roche's time by using the circular restricted three-body model with constant mass. He also used the Gylden-Meshcherskii problem and determined the equilibrium points and the zero velocity surfaces. He observed that the zero velocity surfaces do not exist. Zhang et al. in [61] investigated the triangular libration points in the restricted three-body problem of variable mass in which both the primaries are radiating as well and the infinitesimal body varies its mass with time according to Jean's law and observed that the motion around the triangular libration points are unstable. Ceccaroni et al. in [20] explored the stability of the circular restricted four-body problem. Using this model, they explored the low-thrust propulsion capabilities to generate surfaces of artificial equilibrium points near the smaller primaries, both in and out of plane. Suraj et al. in [44] studied the Sitnokov problem by considering all the three primaries as oblate bodies in the circular restricted four-body problem. They found the stability region of the motion depending on the oblateness parameter by using Floquet theory. Kumari et al. in [31] studied the circular restricted four-body problem by taking two primaries as oblate and of equal masses. They obtained two collinear and six non-collinear equilibrium points. The non-collinear equilibrium points are stable for the particular interval of the mass parameter. They also have demonstrated the convergence as well as the divergence of the equilibrium points by the construction of Newton-Raphson basin of attraction. Asique et al. in [11] investigated the locations and stability of the equilibrium points in the circular restricted four-body problem and observed that the collinear libration points are unstable while the non-collinear equilibration points are stable for the different mass parameter and oblateness factor. They showed that the presence of oblateness coefficient and various values of Jacobi constant expanded the stability regions of the equilibrium points. Falaye in [23] investigated the stability of equilibrium points in the circular restricted four-body problem 
with the effect of oblateness, radiation, and a circular cluster of material points. After considering two equal masses, he obtained that all the equilibrium points are unstable. Papadauris et al. in [42, 43] studied numerically the restricted four-body problem by considering the bigger primary as source of radiation and the other two primaries are equal. They observed that the equilibrium points are depending on the mass parameter and source of radiation. They also illustrated the periodic orbits, the Poincare surface of section and their stability under the effect of radiation pressure. Abouelmagd in [3-6] explored the restricted three-body problem with different perturbations. Papadakis in $[40,41]$ studied the 3D symmetric periodic orbits of the circular restricted four-body problem, through their bifurcation from the plane orbits. He illustrated the characteristic curves and stability diagram of families of 3D periodic orbits. Additionally, he described a grid method to obtain initial conditions for new asymmetric double-periodic orbits. Mittal et al. in [38] investigated the stability of the Lagrangian solutions for the restricted four-body problem with variable mass. They found that all the equilibrium points are unstable. Ansari in $[7,8]$ studied the stability of the equilibrium points in the circular restricted four-body problem with one of the primaries is taken as oblate spheroid and the mass of the infinitesimal body is taken as variable mass body. He found eight equilibrium points in which three are asymptotically stable and the five others are unstable. Ansari in [9] explored the equilibrium points, time series, zero-velocity curves, and Poincare surface of section numerically in the circular restricted three-body problem by considering one of the primaries as oblate spheroid, a second one as a source of radiation pressure and such that all the three bodies vary their masses with time. He also examined the stability of the equilibrium points and found that all the equilibrium points are unstable. Another aspect in relation with our work is the basin of attraction. In this topic, many scientists have studied and determined the basins of attraction. Among them, we can cite for example the works of Douskos [22], Kumari et al. in [31], de Assis and Terra in [21], Matthies et al. in [35], Paricio in [26], Asique et al. in [12], Zotos in [62-65], etc.

Taking in consideration the cited literature, we have studied the effect of coriolis and centrifugal forces in the circular restricted four-body problem by considering all the masses vary with time and one of the primaries as oblate body. The organization of the present paper is as follows: In the introduction section, we have reviewed all the literature related to the problem. In the equations of motion section, we have evaluated the equations of motion with the effect of coriolis and centrifugal forces. In the numerical computations section, we have determined the locations of equilibrium points, surfaces of the motion of the infinitesimal body, time series, and the basins of attraction. In the stability section, we have examined the stability of the equilibrium points. Finally, in the last section, we made some necessary comments about the present work to explain our point of view on the obtained results in view of the classical ones. We are convinced that the results obtained in this study have practical application in astrophysics.

\section{Equation of motion}

In this section, we evaluate the equations of motion of the infinitesimal body with mass $m$ by considering that the three primaries have respectively $m_{1}, m_{2}$, and $m_{3}$ as masses and are placed at the vertices of a triangle. The primary $m_{2}$ is supposed to be an oblate body with oblateness factor $\sigma$ and the masses of all the four bodies are varying with time. The perturbations in the coriolis and centrifugal forces are denoted by $\varphi$ and $\psi$, respectively, the unperturbed value of each being unity. All the primaries are revolving in the circular orbits around their center of mass which is taken as origin. The line, at which the primary $m_{1}$ is placed, is taken as $x$-axis and the line perpendicular to the $x$-axis and passing through the origin is considered as $y$-axis, the line which passes through the origin and perpendicular to the plane of motion of the primaries is taken as $z$-axis (Fig. 1). Let us consider the synodic coordinate system, initially coincide with the inertial coordinate system, and revolve with angular velocity $\omega$ about $z$-axis. Due to oblateness factor s, equilateral triangular configuration with side $\ell$, will no longer exist as a classical case. Let us suppose, in our case, $A B=\ell+\lambda_{1}, B C=\ell+\lambda_{2}, C A=\ell+\lambda_{3}$, where $\lambda_{1}<1, \lambda_{2}<1$ and $\lambda_{3}<1$. Proceeding as in Ansari [7], so we get the following 


$$
\lambda_{1}-\lambda_{3}=\frac{\sigma}{2 \ell}, \lambda_{1}=\lambda_{2} \text {, and } \omega^{2}=\frac{G\left(m_{1}+m_{2}+m_{3}\right)}{\ell^{3}}\left[1-\frac{3}{\ell}\left(\lambda_{1}-\frac{\sigma}{2 \ell}\right)\right] .
$$

This means that the three bodies remain at the vertices of an isosceles triangle instead of an equilateral triangle as in the classical case. Following the procedure of Abdullah [9] and using the Meshcherskii transformation with $(i=1,2,3)$ given by

$$
\left\{\begin{array}{l}
x=\xi R(t), y=\eta R(t), z=\zeta R(t), \frac{d t}{d \tau}=R^{2}(t), r_{i}=\rho_{i} R(t), \\
\omega(t)=\frac{\omega_{0}}{R^{2}(t)}, x_{i}=\xi_{i} R(t), y_{i}=\eta_{i} R(t), v(t)=\frac{v_{0}}{R(t)}, v_{i}(t)=\frac{v_{i 0}}{R(t)}, \\
m=\frac{m_{0}}{R(t)}, R(t)=\sqrt{a t^{2}+2 b t+c}, a c-b^{2}=1-k,
\end{array}\right.
$$

where $k, a, b, c, v_{0}, v_{10}, v_{20}, v_{30}, m_{0}$ are constants.

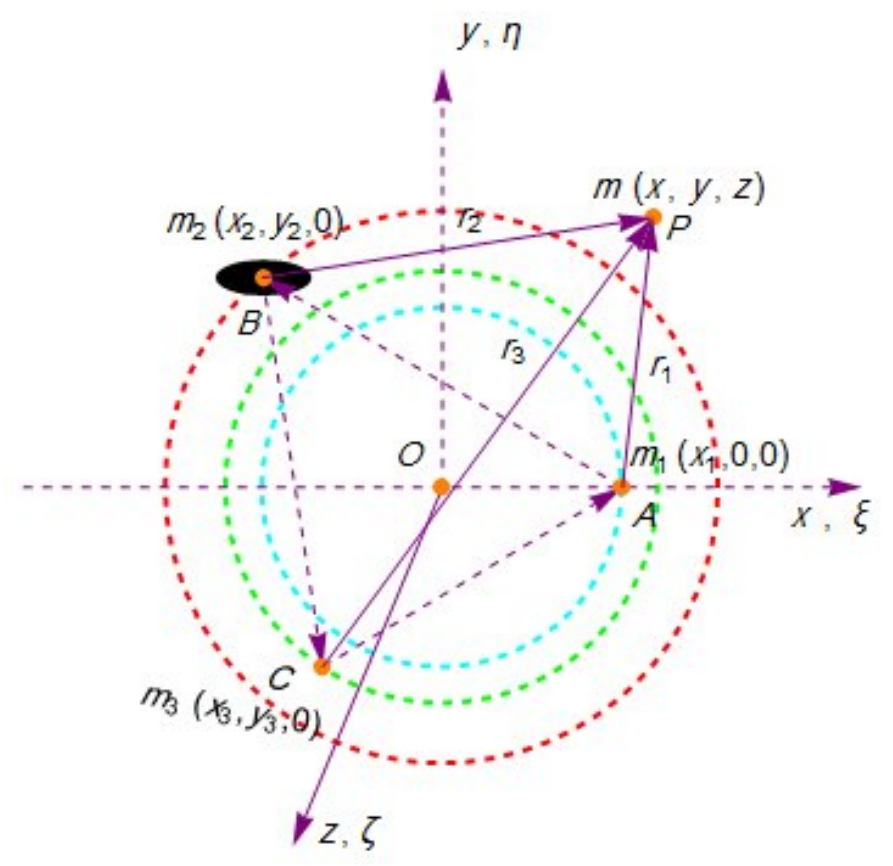

Figure 1: The configuration of the restricted three-body problem with perturbations.

By considering unit of mass, distance and time at initial time $t_{0}$ such that $v_{0}=1, \ell=1, G=1$, $\mathrm{at}_{0}+\mathrm{b}=\alpha_{1}$ (constant), and introducing the mass parameter as

$$
v_{10}=v, v_{20}=\left(1-v-\alpha_{2} v\right), v_{30}=\alpha_{2} v, \quad \alpha_{2} \ll 1,
$$

we get the equations of motion are then defined by

$$
\left\{\begin{array}{l}
\xi^{\prime \prime}-2 \omega_{0} \varphi \eta^{\prime}-\alpha_{1} \xi^{\prime}=\frac{\partial V}{\partial \xi} \\
\eta^{\prime \prime}+2 \omega_{0} \varphi \xi^{\prime}-\alpha_{1} \eta^{\prime}=\frac{\partial V}{\partial \eta} \\
\zeta^{\prime \prime}-\alpha_{1} \zeta^{\prime}=\frac{\partial V}{\partial \zeta}
\end{array}\right.
$$

where

$$
\begin{aligned}
V= & \frac{1}{2}\left(\alpha_{1}^{2}+\omega_{0}^{2} \psi+k-1\right)\left(\xi^{2}+\eta^{2}\right)+\frac{1}{2}\left(\alpha_{1}^{2}+k-1\right) \zeta^{2}-\alpha_{1} \varphi \xi \eta \\
& +\frac{v}{\rho_{1}}+\frac{\left(1-v-\alpha_{2} v\right)}{\rho_{2}}+\frac{\left(1-v-\alpha_{2} v\right) \sigma}{2 \rho_{2}^{3}}+\frac{\alpha_{2} v}{\rho_{3}},
\end{aligned}
$$




$$
\begin{aligned}
\omega_{0}^{2} & =\left(1-3 \lambda_{1}+\frac{3 \sigma}{2}\right), \quad \rho_{i}^{2}=\left(\xi-\xi_{i}\right)^{2}+\left(\eta-\eta_{i}\right)^{2}+\zeta^{2}, \quad\left(\xi_{1}, \eta_{1}\right)=\left(\frac{1}{\sqrt{3}}\left(1+\frac{\lambda_{1}}{3}\right), 0\right), \\
\left(\xi_{2}, \eta_{2}\right) & =\left(-\frac{1}{2 \sqrt{3}}\left(1+\frac{7 \lambda_{1}}{3}\right), \frac{1}{2}\left(1+\lambda_{1}\right)\right), \quad\left(\xi_{3}, \eta_{3}\right)=\left(-\frac{1}{2 \sqrt{3}}\left(1-\frac{5 \lambda_{1}}{3}\right),-\frac{1}{2}\left(1+\lambda_{1}\right)\right) .
\end{aligned}
$$

The symbol prime (') is the differentiation w.r.t. $\tau$.

\section{Graphs with numerical computations}

In this section, we determine the locations of equilibrium points in the three planes; $(\xi, \eta)$-plane $(\xi, \zeta)$-plane and $(\eta, \zeta)$-plane, the surfaces of the motion of the infinitesimal body, the time series, and the basins of attraction by Newton-Raphson method. The numerical methods used in the present study are similar to those presented in [10, 14-19, 28, 60].

\subsection{Locations of equilibrium points}

We can find the locations of the equilibrium points by solving the following equations for $V_{\xi}=V_{\eta}=$ $\mathrm{V}_{\zeta}=0$,

$$
\begin{aligned}
& \left(\alpha_{1}^{2}+\omega_{0}^{2} \psi+k-1\right) \xi-\alpha_{1} \varphi \eta-\frac{v\left(\xi-\xi_{1}\right)}{\rho_{1}^{3}}-\frac{\left(1-v-\alpha_{2} v\right)\left(\xi-\xi_{2}\right)}{\rho_{2}^{3}} \\
& \quad-\frac{3\left(1-v-\alpha_{2} v\right)\left(\xi-\xi_{2}\right) \sigma}{2 \rho_{2}^{5}}-\frac{\alpha_{2} v\left(\xi-\xi_{3}\right)}{\rho_{3}^{3}}=0, \\
& \left(\alpha_{1}^{2}+\omega_{0}^{2} \psi+k-1\right) \eta-\alpha_{1} \varphi \xi-\frac{v \eta}{\rho_{1}^{3}}-\frac{\left(1-v-\alpha_{2} v\right)\left(\eta-\eta_{2}\right)}{\rho_{2}^{3}} \\
& \quad-\frac{3\left(1-v-\alpha_{2} v\right)\left(\eta-\eta_{2}\right) \sigma}{2 \rho_{2}^{5}}-\frac{\alpha_{2} v\left(\eta-\eta_{3}\right)}{\rho_{3}^{3}}=0, \\
& \left(\alpha_{1}^{2}+k-1\right) \zeta-\frac{v \zeta}{\rho_{1}^{3}}-\frac{\left(1-v-\alpha_{2} v\right) \zeta}{\rho_{2}^{3}}-\frac{3\left(1-v-\alpha_{2} v\right) \sigma \zeta}{2 \rho_{2}^{5}}-\frac{\alpha_{2} v \zeta}{\rho_{3}^{3}}=0 .
\end{aligned}
$$

- $(\xi, \eta)$-plane $k=0.4, \alpha_{1}=0.2, v=0.019, \sigma=0.01, \lambda_{1}=0.01, \alpha_{2}=0.01$.

In this plane, we found five equilibrium points and observed that

1. when we increase both the coriolis and centrifugal forces, the curves are shrinking and the equilibrium points are towards the origin (Fig. 2 (a));

2. when we fix the coriolis force and increase the value of centrifugal force, the curves are shrinking and the equilibrium points are towards the origin (Fig. 2 (b));

3. when we fix the centrifugal force and increase the value of coriolis force, the curves are expanding and the equilibrium points are away from the origin (Fig. 2 (c)).

In Figure 2, we have the color code: $\varphi=\psi=1.2$ (red), $\varphi=\psi=1.4$ (green), and the purple color points indicate the locations of the primaries.

- $(\xi, \zeta)$-plane $k=0.4, \alpha_{1}=0.2, v=0.019, \sigma=0.01, \lambda_{1}=0.01, \alpha_{2}=0.01$.

In this plane, we found five equilibrium points and observed that when we increase the values of the coriolis and centrifugal forces, the curves are shrinking and the equilibrium points are moving towards the origin. See Figure $3(\varphi=\psi=1.2$ (red), $\varphi=\psi=1.4$ (green)).

- $(\eta, \zeta)$-plane $k=0.4, \alpha_{1}=0.2, v=0.019, \sigma=0.01, \lambda_{1}=0.01, \alpha_{2}=0.01$.

In this plane, we found three equilibrium points and observed that when we increase the values of the coriolis and centrifugal forces, the curves are shrinking and the equilibrium points are moving towards the origin. Figure 4 gives more detail ( $\varphi=\psi=1.2$ (red), $\varphi=\psi=1.4$ (green)). 
(a)

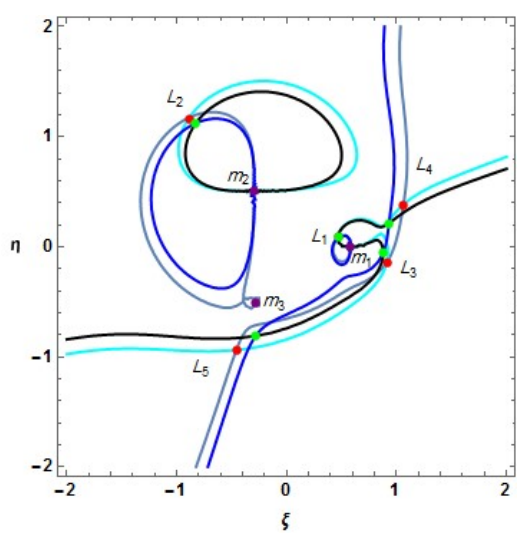

(b)

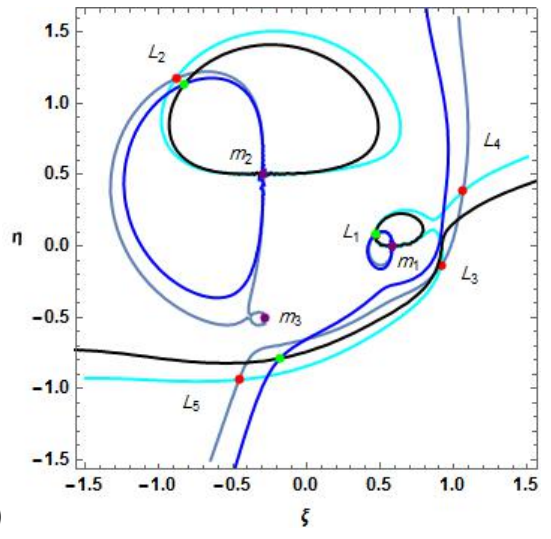

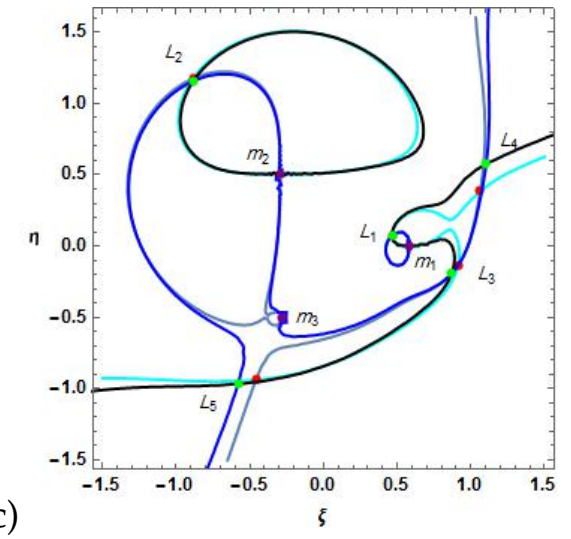

Figure 2: The locations of equilibrium points at (a) : $\varphi=\psi=1.2$ (red), $\varphi=\psi=1.4$ (green). (b) : $\varphi=\psi=1.2$ (red), $\varphi=1.2, \psi=1.4$ (green). (c) : $\varphi=\psi=1.2$ (red), $\varphi=1.4, \psi=1.2$ (green).

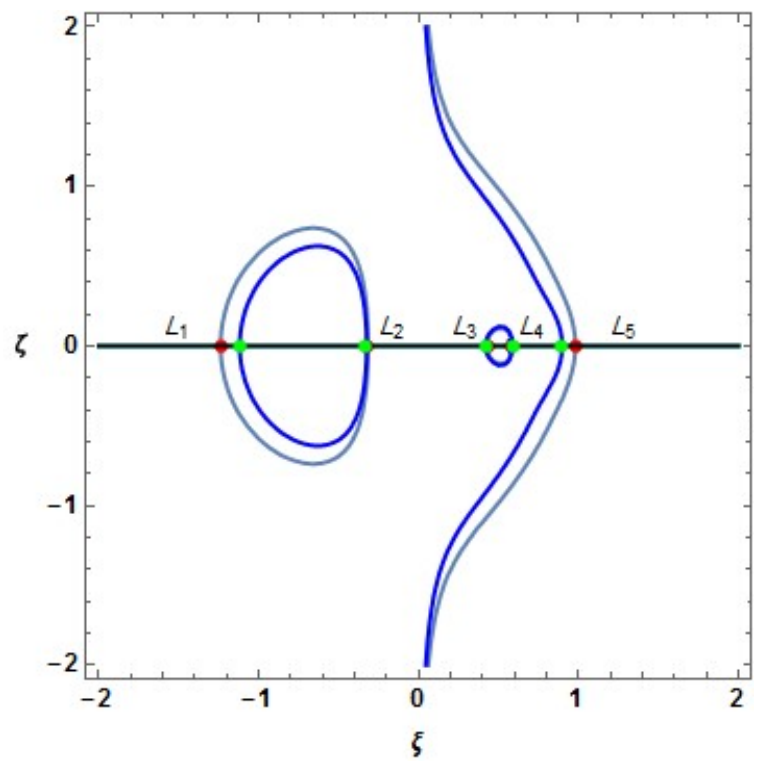

Figure 3: The locations of equilibrium points at $\varphi=\psi=1.2$ (red), $\varphi=\psi=1.4$ (green).

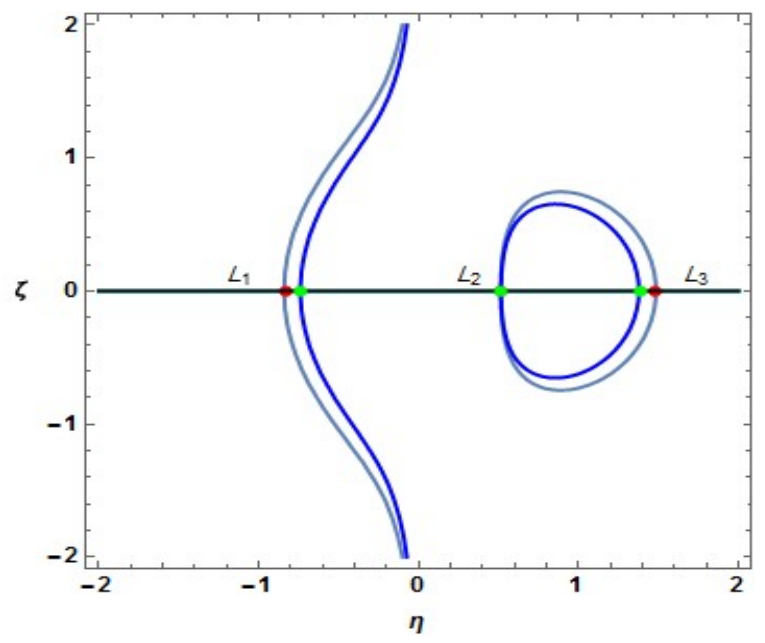

Figure 4: The locations of equilibrium points at $\varphi=\psi=1.2$ (red), $\varphi=\psi=1.4$ (green). 


\subsection{Surfaces}

In this section, we have drawn the surfaces of motion of the infinitesimal body under the effect of the coriolis and centrifugal forces and found that when we increase the values of the coriolis and centrifugal forces, the surfaces are shrinking (Figs. 5 and 6).

(a)

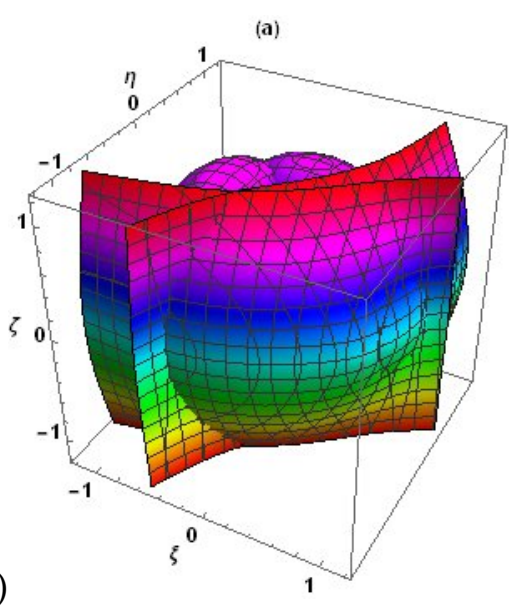

(b)

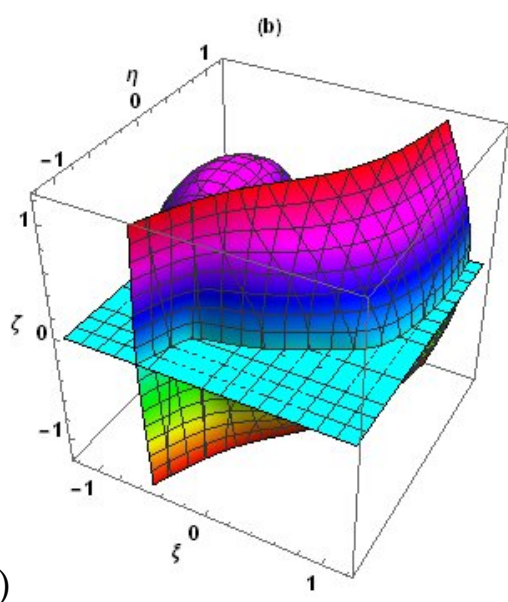

(c)

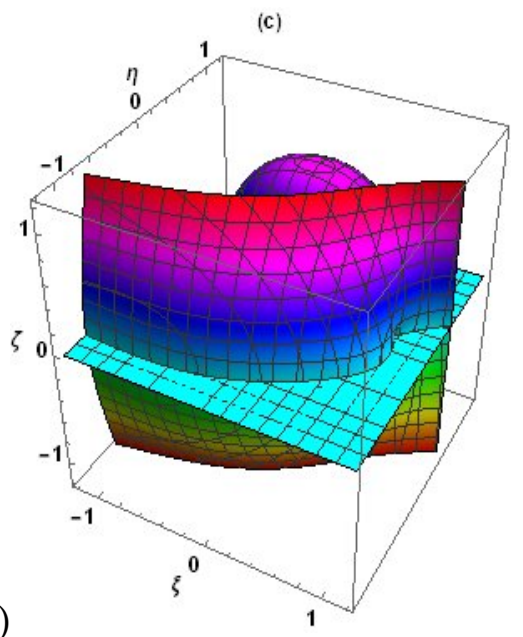

Figure 5: The surfaces of motion of infinitesimal body at $\varphi=\psi=1.2$.

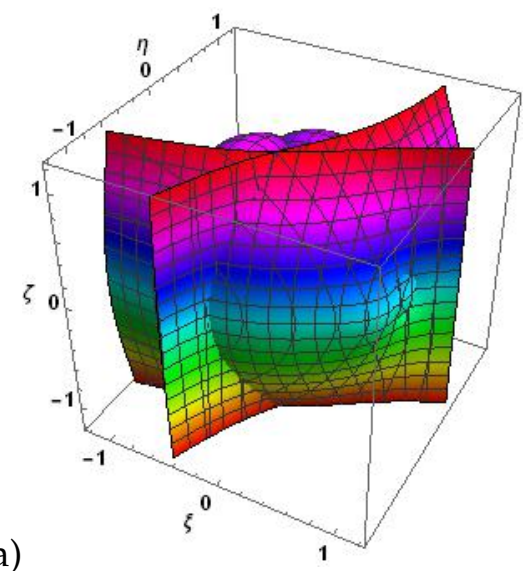

(b) (c)

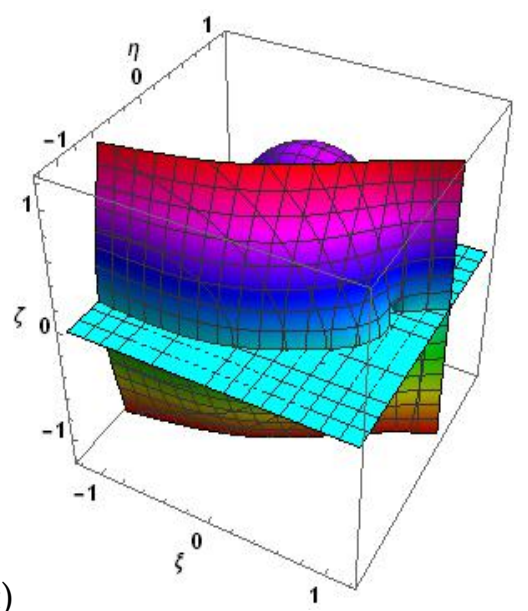

Figure 6: The surfaces of motion of infinitesimal body at $\varphi=\psi=1.4$.

\subsection{Time series}

For this section, we determined the time series in $(\tau, \xi)$ and $(\tau, \eta)$ under the effect of the coriolis and centrifugal forces. We observed that when we increase the values of the coriolis and centrifugal forces, the curves are shifting by some phase angles and also according to the pattern of the curves, we concluded that the orbits will not be periodic (Figs. 7 and 8).

\subsection{Newton-Raphson basins of attraction}

In this section, we drawn the basins of attraction for the circular restricted four-body problem under the effect of perturbations (oblateness, variable of masses and the coriolis and centrifugal forces) by using Newton-Raphson iterative method. It is very fast and accurate computational tool. The algorithm of our problem is given by, 


$$
\left\{\begin{array}{l}
\xi_{n}=\xi_{n-1}-\left(\frac{V_{\xi} V_{\eta \eta}-V_{\eta} V_{\xi \eta}}{V_{\xi \xi} V_{\eta \eta}-V_{\xi \eta} V_{\eta \xi}}\right)_{\left(\xi_{n-1}, \eta_{n-1}\right)^{\prime}}, \\
\eta_{n}=\eta_{n-1}-\left(\frac{V_{\eta} V_{\xi \xi}-V_{\xi} V_{\eta \xi}}{V_{\xi \xi} V_{\eta \eta}-V_{\xi \eta} V_{\eta \xi}}\right)_{\left(\xi_{n-1}, \eta_{n-1}\right)^{\prime}}
\end{array}\right.
$$

where $\xi_{n-1}, \eta_{n-1}$ are the values of the $\xi$ and $\eta$ coordinates of the $(n-1)^{\text {th }}$ step of the Newton-Raphson iterative process. The initial point $(\xi, \eta)$ is a member of the basin of attraction of the root if this point converges rapidly to one of the equilibrium points. This process stops when the successive approximations converge to an attractor with some predefined accuracy. In this process the successive approximation points create a crocked path line. For the classification of the equilibrium points on the $(\xi, \eta)$-plane, we used color code. In this way a complete view of the basin structures created by the attractors (Figs. 9 (a) and 10 (a)). The Figure 9 (a) represents the basin of attraction at $\varphi=\psi=1.2$, where the black sign like spider is the configuration of the primaries. The zoomed Figure 9 (b) of the Figure 9 (a) gives us a better detail of this configuration. The Figure 10 (a) represents the basin of attraction at $\varphi=\psi=1.4$, in which the black sign like spider is the configuration of the primaries. The zoomed Figure $10(\mathrm{~b})$ of the Figure 10 (a), gives us also a better detail on this configuration. We also observed that when we increase the values of the coriolis and centrifugal forces, the basin of attraction is shrinking.

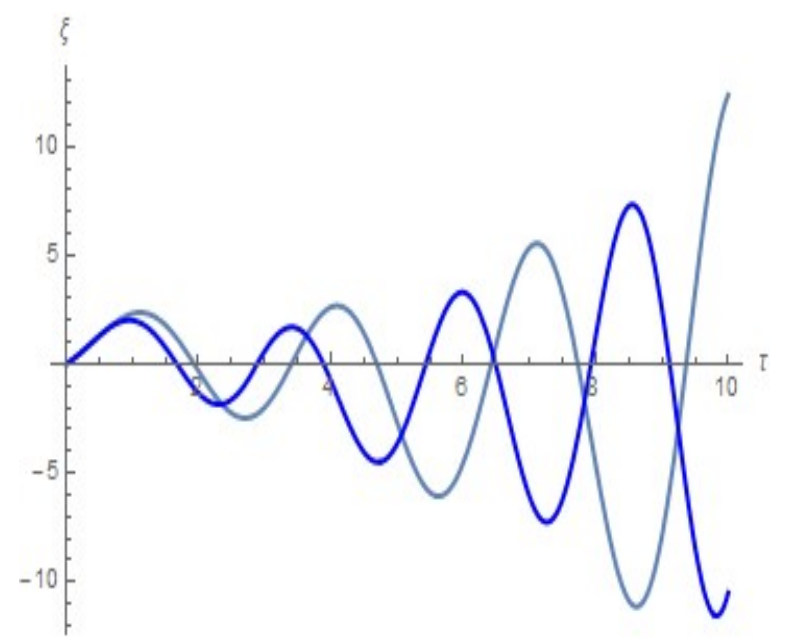

Figure 7: The time series in $(\tau, \xi), \varphi=\psi=1.2$ (magneta), $\varphi=\psi=1.4$ (blue).

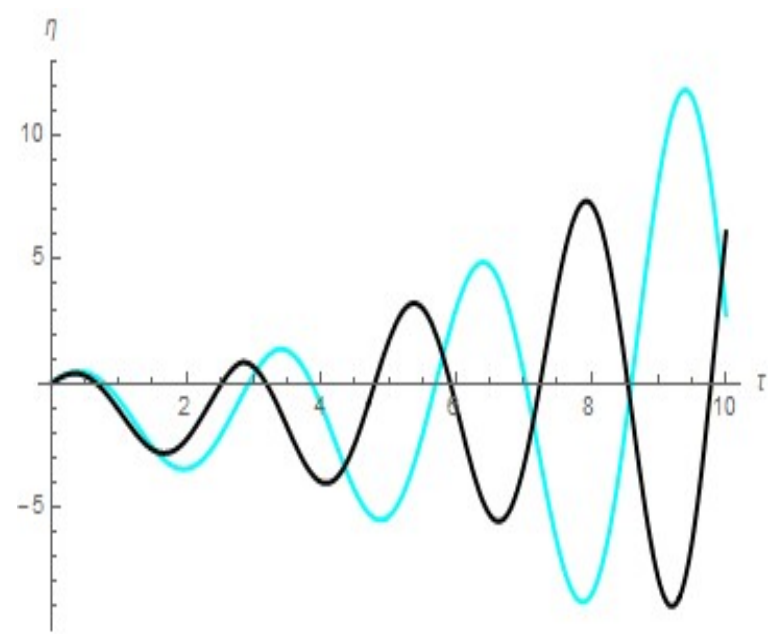

Figure 8: The time series in $(\tau, \eta), \varphi=\psi=1.2$ (cyan), $\varphi=\psi=1.4$ (black). 

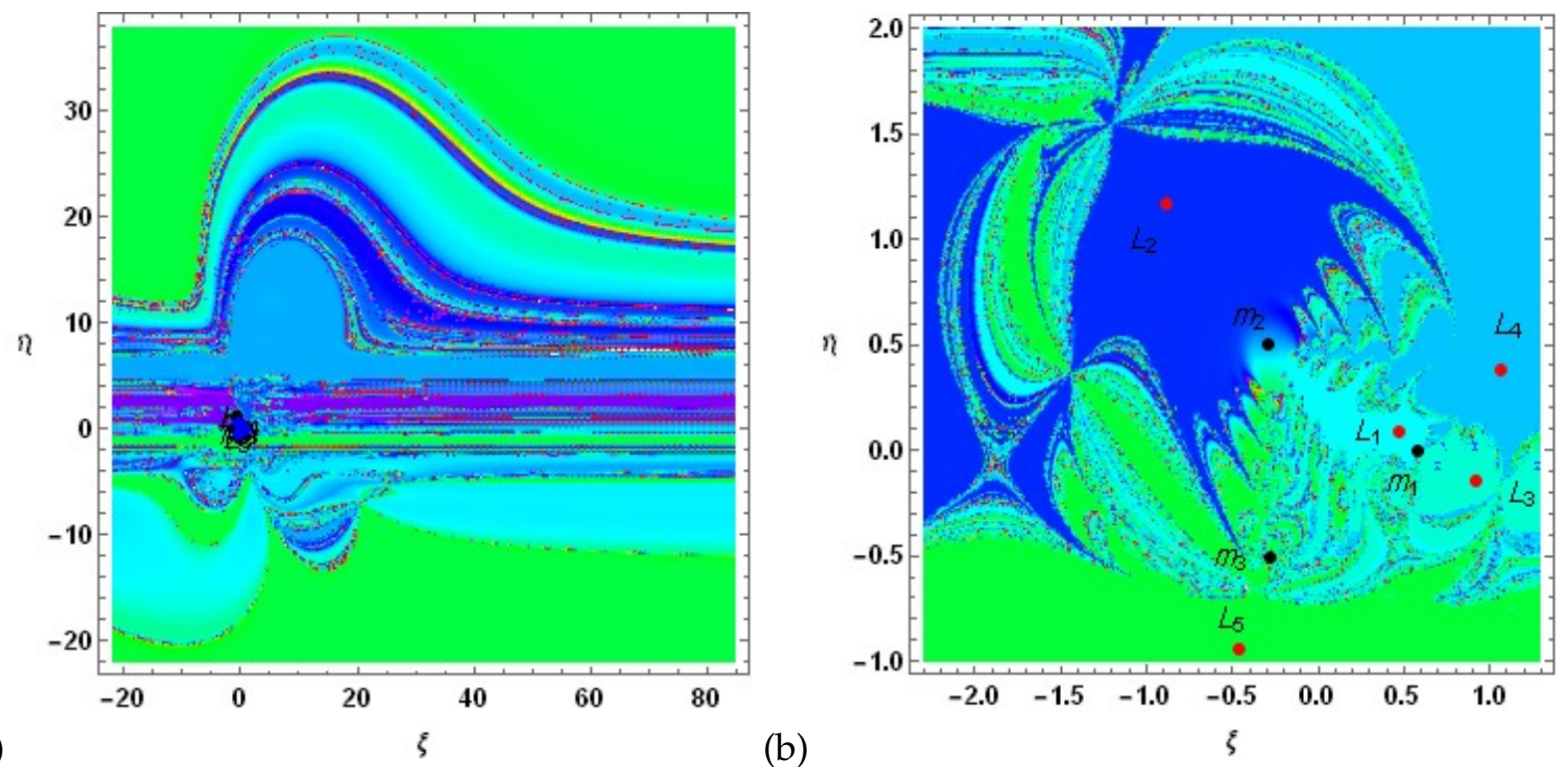

Figure 9: (a): The basin of attractions at $\varphi=\psi=1.2$. (b): The zoomed image near the primaries.
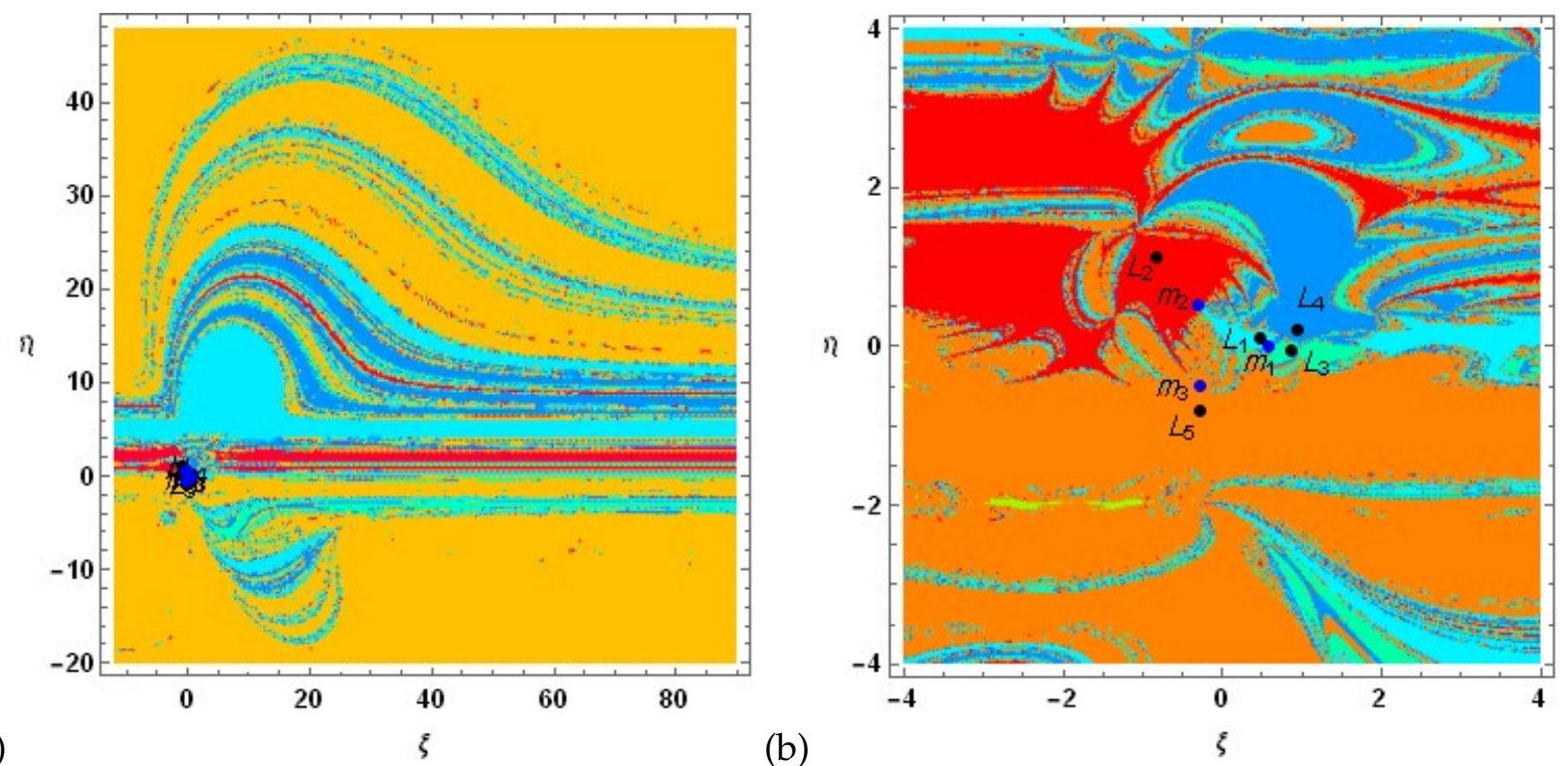

Figure 10: (a): The basin of attractions at $\varphi=\psi=1.4$. (b): The zoomed image near the primaries.

\subsection{Stability of equilibrium points}

Using the procedure given by Mccuskey [36], we have examined the stability of the equilibrium points. By taking $\xi=\xi_{0}+\alpha, \eta=\eta_{0}+\beta, \zeta=\zeta_{0}+\gamma$ in equation (2.1), we get

$$
\left\{\begin{array}{l}
\alpha^{\prime \prime}-2 \varphi \beta^{\prime}-\alpha_{1} \alpha^{\prime}=\alpha\left(V_{\xi \xi}\right)_{0}+\beta\left(V_{\xi \eta}\right)_{0}+\gamma\left(V_{\xi \zeta}\right)_{0} \\
\beta^{\prime \prime}+2 \varphi \alpha^{\prime}-\alpha_{1} \beta^{\prime}=\alpha\left(V_{\eta \xi}\right)_{0}+\beta\left(V_{\eta \eta}\right)_{0}+\gamma\left(V_{\eta \zeta}\right)_{0} \\
\gamma^{\prime \prime}-\alpha_{1} \gamma^{\prime}=\alpha\left(V_{\zeta \xi}\right)_{0}+\beta\left(V_{\zeta \eta}\right)_{0}+\gamma\left(V_{\zeta \zeta}\right)_{0}
\end{array}\right.
$$

where $\alpha, \beta$ and $\gamma$ are the small displacements of the infinitesimal body from the equilibrium point. Suffix zero denotes the value at the equilibrium point.

To solve equation (3.1), let $\alpha=A e^{\lambda \tau}, \beta=B e^{\lambda \tau}, \gamma=C e^{\lambda \tau}$, where $A, B$, and $C$ are parameters. Substi- 
tuting these values in equation (3.1) and rearranging, we get

$$
\left\{\begin{array}{l}
\mathrm{A}\left(\lambda^{2}-\alpha_{1} \lambda-\left(\mathrm{V}_{\xi \xi}\right)_{0}\right)-\mathrm{B}\left(2 \varphi \lambda+\left(\mathrm{V}_{\xi \eta}\right)_{0}\right)-\mathrm{C}\left(\mathrm{V}_{\xi \zeta}\right)_{0}=0 \\
\mathrm{~A}\left(2 \varphi \lambda-\left(\mathrm{V}_{\eta \xi}\right)_{0}\right)+\mathrm{B}\left(\lambda^{2}-\alpha_{1} \lambda-\left(\mathrm{V}_{\eta \eta}\right)_{0}\right)-C\left(\mathrm{~V}_{\eta \zeta}\right)_{0}=0 \\
-\mathrm{A}\left(\mathrm{V}_{\zeta \xi}\right)_{0}-\mathrm{B}\left(\mathrm{V}_{\zeta \eta}\right)_{0}+\mathrm{C}\left(\lambda^{2}-\alpha_{1} \lambda-\left(\mathrm{V}_{\zeta \zeta}\right)_{0}\right)=0
\end{array}\right.
$$

The equation (3.2) will have a non-trivial solution for $A, B$, and $C$ if

$$
\begin{aligned}
& \left|\begin{array}{ccc}
\lambda^{2}-\alpha_{1} \lambda-\left(\mathrm{V}_{\xi \xi}\right)_{0} & -\left(2 \varphi \lambda+\left(\mathrm{V}_{\xi \eta}\right)_{0}\right) & -\left(\mathrm{V}_{\xi \zeta}\right)_{0} \\
2 \varphi \lambda+\left(\mathrm{V}_{\eta \xi}\right)_{0} & \lambda^{2}-\alpha_{1} \lambda-\left(\mathrm{V}_{\eta \eta}\right)_{0} & -\left(\mathrm{V}_{\eta \zeta}\right)_{0} \\
-\left(\mathrm{V}_{\zeta \xi}\right)_{0} & -\left(\mathrm{V}_{\zeta \eta}\right)_{0} & \lambda^{2}-\alpha_{1} \lambda-\left(\mathrm{V}_{\zeta \zeta}\right)_{0}
\end{array}\right|=0 \Longleftrightarrow \\
& \lambda^{6}-3 \alpha_{1} \lambda^{5}+\lambda^{4}\left(4 \varphi^{2}+3 \alpha_{1}^{2}-\left(\mathrm{V}_{\xi \xi}\right)_{0}-\left(\mathrm{V}_{\eta \eta}\right)_{0}-\left(\mathrm{V}_{\zeta \zeta}\right)_{0}\right)+\alpha_{1} \lambda^{3}\left(-4 \varphi^{2}-\alpha_{1}^{2}+2\left(\mathrm{~V}_{\xi \xi}\right)_{0}\right. \\
& \left.\quad+2\left(\mathrm{~V}_{\eta \eta}\right)_{0}+2\left(\mathrm{~V}_{\zeta \zeta}\right)_{0}\right)+\lambda^{2}\left(-\left(\mathrm{V}_{\xi \eta}\right)_{0}^{2}-\left(\mathrm{V}_{\xi \zeta}\right)_{0}^{2}+\left(\mathrm{V}_{\xi \xi}\right)_{0}\left(\mathrm{~V}_{\eta \eta}\right)_{0}-\left(\mathrm{V}_{\eta \zeta}\right)_{0}^{2}-4 \varphi^{2}\left(\mathrm{~V}_{\zeta \zeta}\right)_{0}\right. \\
& \left.\quad+\left(\mathrm{V}_{\xi \xi}\right)_{0}\left(\mathrm{~V}_{\zeta \zeta}\right)_{0}+\left(\mathrm{V}_{\zeta \zeta}\right)_{0}\left(\mathrm{~V}_{\eta \eta}\right)_{0}-\alpha_{1}^{2}\left(\mathrm{~V}_{\xi \xi}\right)_{0}-\alpha_{1}^{2}\left(\mathrm{~V}_{\eta \eta}\right)_{0}-\alpha_{1}^{2}\left(\mathrm{~V}_{\zeta \zeta}\right)_{0}\right) \\
& \quad+\alpha_{1} \lambda\left(\left(\mathrm{V}_{\xi \eta}\right)_{0}^{2}+\left(\mathrm{V}_{\xi \zeta}\right)_{0}^{2}-\left(\mathrm{V}_{\xi \xi}\right)_{0}\left(\mathrm{~V}_{\eta \eta}\right)_{0}+\left(\mathrm{V}_{\eta \zeta}\right)_{0}^{2}-\left(\mathrm{V}_{\xi \xi \xi}\right)_{0}\left(\mathrm{~V}_{\zeta \zeta \zeta}\right)_{0}-\left(\mathrm{V}_{\eta \eta}\right)_{0}\left(\mathrm{~V}_{\zeta \zeta}\right)_{0}\right) \\
& \quad+\left(\left(\mathrm{V}_{\xi \zeta}\right)_{0}^{2}\left(\mathrm{~V}_{\eta \eta}\right)_{0}-2\left(\mathrm{~V}_{\xi \eta}\right)_{0}\left(\mathrm{~V}_{\xi \zeta}\right)_{0}\left(\mathrm{~V}_{\eta \zeta}\right)_{0}+\left(\mathrm{V}_{\xi \xi}\right)_{0}\left(\mathrm{~V}_{\eta \zeta}\right)_{0}^{2}+\left(\mathrm{V}_{\xi \eta}\right)_{0}^{2}\left(\mathrm{~V}_{\zeta \zeta}\right)_{0}\right. \\
& \left.\quad-\left(\mathrm{V}_{\xi \xi}\right)_{0}\left(\mathrm{~V}_{\eta \eta}\right)_{0}\left(\mathrm{~V}_{\zeta \zeta}\right)_{0}\right)=0 .
\end{aligned}
$$

We solved the equation (3.3) for the different values of the equilibrium points and found that in all the cases, among the values of $\lambda$, some of them are positive real values and the others are complex values with non-null imaginary part. Hence, all the equilibrium points are unstable.

\section{Conclusion}

In this paper, we have investigated the stability of the equilibrium points in the circular restricted four-body problem under the effect of oblateness of one of the primaries, variation of masses, coriolis and centrifugal forces. Due to oblateness, we found that the equilateral triangular configuration will no longer exist but it will become an isosceles triangular. On the other hand under these variations, the equations of motion differ by $\sigma, \alpha_{1}, k, \varphi$, and $\psi$, from the equations of motion in the classical case. We have drawn the location of the equilibrium points in the three planes, $(\xi, \eta)$-plane, $(\xi, \zeta)$-plane and $(\eta, \zeta)$-plane, and observed that the equilibrium points are depending on both the coriolis and centrifugal forces which also differs from the classical case since in this case it depends only on the centrifugal force. In both the $(\xi, \eta)$-plane and $(\xi, \zeta)$-plane, we found five equilibrium points (Figs. 2 and 3). However, in the $(\eta, \zeta)$-plane, we found three equilibrium points (Fig. 4). In all these planes, we observed that, when we increased the values of the coriolis and centrifugal forces, the curves are shrinking and the equilibrium points are towards the origin except when we increased the coriolis force and fixed the centrifugal force, the equilibrium points are away from the origin (Fig. 2 (c)). We have drawn the surfaces of the motion of the infinitesimal body and observed that when we increased the values of the coriolis and centrifugal forces, the curves are shrinking (Figs. 5 and 6). We also have drawn the time series (Figs. 7 and 8 ) and observed that when we increase the values of the coriolis and centrifugal forces, the curves are shifting by some phase angles and the pattern of the curves show that the orbits will not be periodic. As an important part of the study, we have drawn the Newton-Raphson basins of attraction (Figs. 9 (a) and 10 (a)). The Figure 9 (a) is at $\varphi=\psi=1.2$, and the Figure 10 (a) is at $\varphi=\psi=1.4$. In the Figures 9 (a) and 10 (a), the black color spider shape shows the configuration of the primaries which can be seen in the zoomed Figure 9 (b) of the Figure 9 (a) and in the zoomed Figure 10 (b) of Figure 10 (a). We observed that when we increased the values of the coriolis and centrifugal forces, the basin of attraction is shrinking. As a final deal, we have examined the stability of the equilibrium points and found that all the equilibrium points are unstable. 


\section{Acknowledgment}

The authors extend their appreciation to the Deanship of Scientific Research at Majmaah University for funding this work through research project No. 37/92.

\section{References}

[1] A. R. AbdulRaheem, J. Singh, Combined effects of perturbations, radiation, and oblateness on the stability of equilibrium points in the restricted three-body problem, Astron. J., 131 (2006), 1880-1885. 1

[2] A. R. AbdulRaheem, J. Singh, Combined effects of perturbations, radiation, and oblateness on the periodic orbits in the restricted three-body problem, Astrophys. Space Sci., 317 (2008), 9-13. 1

[3] E. I. Abouelmagd, H. M. Asiri, M. A. Sharaf, The effect of oblateness in the perturbed restricted three-body problem, Meccanica, 48 (2013), 2479-2490. 1

[4] E. I. Abouelmagd, S. M. El-Shaboury, Periodic orbits under combined effects of oblateness and radiation in the restricted problem of three bodies, Astrophys. Space Sci., 341 (2012), 331-341.

[5] E. I. Abouelmagd, J. L. G. Guirao, On the perturbed restricted three-body problem, Appl. Math. Nonlinear Sci., 1 (2016), 123-144.

[6] E. I. Abouelmagd, A. Mostafa, Out of plane equilibrium points locations and the forbidden movement regions in the restricted three-body problem with variable mass, Astrophys. Space Sci., 357 (2015), 10 pages. 1

[7] A. A. Ansari, Stability of the equilibrium points in the circular restricted four body problem with oblate primary and variable mass, Int. J. Adv. Astron., 4 (2016), 14-19. 1, 2

[8] A. A. Ansari, Stability of the Libration points in the photogravitational circular restricted four-body problem with the effect of perturbations variable mass, Sci. Int. (Lahore), 28 (2016), 859-866. 1

[9] A. A. Ansari, Dynamics in the circular restricted three body problem with perturbations, Int. J. Adv. Astron., 5 (2017), 19-25. 1, 2

[10] A. A. Ansari, R. Kellil, Periodic motion of Martian moon's satellite, Nonlinear Sci. Lett. A, 8 (2017), 171-177. 3

[11] M. C. Asique, U. Prasad, M. R. Hassan, M. S. Suraj, On the R4BP when third primary is an oblate spheroid, Astrophys. Space Sci., 357 (2015), 13 pages. 1

[12] M. C. Asique, U. Prasad, M. R. Hassan, M. S. Suraj, On the photogravitational R4BP when the third primary is a triaxial rigid body, Astrophys. Space Sci., 361 (2016), 18 pages. 1

[13] K. B. Bhatnagar, P. P. Hallan, Effect of perturbations in coriolis and centrifugal forces on the stability of libration points in the restricted problem, Celestial Mech., 18 (1978), 105-112. 1

[14] M. M. Bhatti, T. Abbas, M. M. Rashidi, A new numerical simulation of MHD stagnation-point flow over a permeable stretching/shrinking sheet in porous media with heat transfer, Iran. J. Sci. Technol. Trans. A Sci., 2016 (2016), 7 pages. 3

[15] M. M. Bhatti, T. Abbas, M. M. Rashidi, M. S. Ali, Numerical simulation of entropy generation with thermal radiation on MHD Carreau nanofluid towards a shrinking sheet, Entropy, 18 (2016), 14 pages.

[16] M. M. Bhatti, T. Abbas, M. M. Rashidi, M. S. Ali, Z.-G. Yang, Entropy generation on MHD Eyring-Powell nanofluid through a permeable stretching surface, Entropy, 18 (2016), 14 pages.

[17] M. M. Bhatti, S. R. Mishra, T. Abbas, M. M. Rashidi, A mathematical model of MHD nanofluid flow having gyrotactic microorganisms with thermal radiation and chemical reaction effects, Neural Comput. Appl., 2016 (2016), 13 pages.

[18] M. M. Bhatti, M. M. Rashidi, Effects of thermo-diffusion and thermal radiation on Williamson nanofluid over a porous shrinking/stretching sheet, J. Mol. Liq., 221 (2016), 567-573.

[19] M. M. Bhatti, M. M. Rashidi, Numerical simulation of entropy generation on MHD nanofluid towards a stagnation point flow over a stretching surface, Int. J. Appl. Comput. Math., 2016 (2016), 15 pages. 3

[20] M. Ceccaroni, J. Biggs, Low-thrust propulsion in a coplanar circular restricted four body problem, Celestial Mech. Dynam. Astronom., 112 (2012), 191-219. 1

[21] S. C. de Assis, M. O. Terra, Escape dynamics and fractal basin boundaries in the planar Earth-Moon system, Celestial Mech. Dynam. Astronom., 120 (2014), 105-130. 1

[22] C. N. Douskos, Collinear equilibrium points of Hills problem with radiation and oblateness and their fractal basins of attraction, Astrophys. Space Sci., 326 (2010), 263-271. 1

[23] B. J. Falaye, Effect of oblateness, radiation and a circular cluster of material points on the stability of equilibrium points in the restricted four-body problem, Few-Body Syst., 56 (2015), 29-40. 1

[24] P. P. Hallan, K. B. Magang, Effect of perturbations in coriolis and centrifugal forces on the nonlinear stability of equilibrium point in robe's restricted circular three-body problem, Adv. Astron., 2008 (2008), 21 pages. 1

[25] P. P. Hallan, N. Rana, Effect of perturbations in coriolis and centrifugal forces on the location and stability of the equilibrium point in the Robe's circular restricted three body problem, Planet. Space Sci., 49 (2001), 957-960. 1

[26] L. J. Hernández Paricio, Bivariate Newton-Raphson method and toroidal attraction basins, Numer. Algorithms, 71 (2016), 349-381. 1

[27] J. H. Jeans, Astronomy and cosmogony, Cambridge University Press, Cambridge, (1929). 1

[28] A. A. Khan, S. Muhammad, R. Ellahi, Q. M. Z. Zia, Bionic study of variable viscosity on MHD peristaltic flow of Pseudoplastic fluid in an asymmetric channel, J. Magn., 21 (2016), 273-280. 3 
[29] M. Khanna, K. B. Bhatnagar, Existence and stability of libration points in the restricted three body problem when the smaller primary is a triaxial rigid body, Indian J. Pure Appl. Math., 29 (1998), 1011-1023. 1

[30] M. Khanna, K. B. Bhatnagar, Existence and stability of libration points in the restricted three body problem when the smaller primary is a triaxial rigid body and the bigger one an oblate spheroid, Indian J. Pure Appl. Math., 30 (1999), 721-733. 1

[31] R. Kumari, B. S. Kushvah, Stability regions of equilibrium points in restricted four-body problem with oblateness effects, Astrophys. Space Sci., 349 (2014), 693-704. 1

[32] E. S. G. Leandro, On the central configurations of the planar restricted four-body problem, J. Differential Equations, 226 (2006), 323-351. 1

[33] L. G. Luk'yanov, On the restricted circular conservative three-body problem with variable masses, Astron. Lett., 35 (2009), 349-359. 1

[34] A. Majorana, On a four-body problem, Celestial Mech. Dynam. Astronom., 25 (1981), 267-270. 1

[35] G. Matthies, M. Salimi, S. Sharifi, J. L. Varona, An optimal three-point eighth-order iterative method without memory for solving nonlinear equations with its dynamics, Jpn. J. Ind. Appl. Math., 33 (2016), 751-766. 1

[36] S. W. McCuskey, Introduction to celestial mechanics, Addisson-Wesley, New York, (1963). 3.5

[37] I. V. Meščerskiǐ, Raboty po mehanike tel peremennormassy, (Russian) [[Works on the mechanics of bodies of variable mass.]] 2d ed., Gosudarstv. Izdat. Tehn.-Teor. Lit., Moscow, (1952), 280 pages. 1

[38] A. Mittal, R. Aggarwal, M. S. Suraj, V. S. Bisht, Stability of libration points in the restricted four-body problem with variable mass, Astrophys. Space Sci,. 361 (2016), 14 pages. 1

[39] F. R. Moulton, On a class of particular solutions of the problem of four bodies, Trans. Amer. Math. Soc., 1 (1900), 17-29. 1

[40] K. E. Papadakis, Families of asymmetric periodic solutions in the restricted four-body problem, Astrophys. Space Sci., 361 (2016), 15 pages. 1

[41] K. E. Papadakis, Families of three-dimensional periodic solutions in the circular restricted four-body problem, Astrophys. Space Sci., 361 (2016), 14 pages. 1

[42] J. P. Papadouris, K. E. Papadakis, Equilibrium points in the photogravitational restricted four-body problem, Astrophys. Space Sci., 344 (2013), 21-38. 1

[43] J. P. Papadouris, K. E. Papadakis, Periodic solutions in the photogravitational restricted four-body problem, Mon. Not. R. Astron. Soc., 442 (2014), 1628-1639. 1

[44] M. Sanam Suraj, M. R. Hassan, Solution of Sitnikov restricted four body problem when all the primaries are oblate bodies: circular case, Proc. Pakistan Acad. Sci., 50 (2013), 61-79. 1

[45] R. K. Sharma, P. V. S. Rao, Collinear equilibria and their characteristic exponents in the restricted three-body problem when the primaries are oblate spheroids, Celestial Mech. Dynam. Astronom., 12 (1975), 189-201. 1

[46] A. K. Shrivastava, D. Garain, Effect of perturbation on the location of libration point in the Robe restricted problem of three bodies, Celestial Mech. Dynam. Astronom., 51 (1991), 67-73. 1

[47] A. K. Shrivastava, B. Ishwar, Equations of motion of the restricted problem of three bodies with variable mass, Celestial Mech., 30 (1983), 323-328. 1

[48] S.-H. Shu, B.-K. Lu, Effect of perturbation of coriolis and Centrifugal forces on the location and linear stability of the libration points in the Robe problem, Chin. Astron. Astrophys., 29 (2005), 421-429. 1

[49] C. Simó, Relative equilibrium solutions in the four-body problem, Celestial Mech., 18 (1978), 165-184. 1

[50] J. Singh, Photogravitational restricted three-body problem with variable mass, Indian J. Pure Appl. Math., 34 (2003), 335-341. 1

[51] J. Singh, Combined effects of oblateness and radiation on the nonlinear stability of $\mathrm{L}_{4}$ in the restricted three-body problem, Astron. J., 137 (2009), 3286-3292.

[52] J. Singh, B. Ishwar, Effect of perturbations on the location of equilibrium points in the restricted problem of three bodies with variable mass, Celestial Mech., 32 (1984), 297-305.

[53] J. Singh, B. Ishwar, Effect of perturbations on the stability of triangular points in the restricted problem of three bodies with variable mass, Celestial Mech. Dynam. Astronom., 35 (1985), 201-207.

[54] J. Singh, O. Leke, Stability of the photogravitational restricted three-body problem with variable masses, Astrophys. Space Sci., 326 (2010), 305-314.

[55] J. Singh, O. Leke, Robe's restricted three-body problem with variable masses and perturbing forces, ISRN Astron. Astrophys., 2013 (2013), 8 pages.

[56] J. Singh, A. Umar, Effect of oblateness of an artificial satellite on the orbits around the triangular points of the Earth-Moon system in the axisymmetric ER3BP, Differ. Equ. Dyn. Syst., 25 (2017), 11-27.

[57] J. Singh, A. E. Vincent, Effect of perturbations in the coriolis and centrifugal forces on the stability of equilibrium points in the restricted four-body problem, Few-Body Syst., 56 (2015), 713-723.

[58] J. Singh, A. E. Vincent, Equilibrium points in the restricted four-body problem with radiation pressure, Few-Body Syst., 57 (2016), 83-91.

[59] J. Singh, A. E. Vincent, Out-of-plane equilibrium points in the photogravitational restricted four-body problem with oblateness, British J. Math. Comput. Sci., 19 (2016), 1-15. 1

[60] K. Vafai, A. A. Khan, S. Sajjad, R. Ellahi, The study of peristaltic motion of third grade fluid under the effects of Hall current and heat transfer, Z. Naturforsch. A, 70 (2015), 281-293. 3 
[61] M.-J. Zhang, C.-Y. Zhao, Y.-Q. Xiong, On the triangular libration points in photogravitational restricted three-body problem with variable mass, Astrophys. Space Sci., 337 (2012), 107-113. 1

[62] E. E. Zotos, Fractal basin boundaries and escape dynamics in a multiwell potential, Nonlinear Dynam., 85 (2016), 16131633. 1

[63] E. E. Zotos, Fractal basins of attraction in the planar circular restricted three-body problem with oblateness and radiation pressure, Astrophys. Space Sci., 181 (2016), 17 pages.

[64] E. E. Zotos, Investigating the Newton-Raphson basins of attraction in the restricted three-body problem with modified Newtonian gravity, J. Appl. Math. Comput., 2016 (2016), 19 pages.

[65] E. E. Zotos, Revealing the basins of convergence in the planar equilateral restricted four-body problem, Astrophys. Space Sci., 362 (2017), 19 pages. 1 